\title{
Comparison Solutions Between Lie Group Method and Numerical Solution of (RK4) for Riccati Differential Equation
}

\author{
Sami H. Altoum ${ }^{1}$, Salih Y. Arbab ${ }^{2}$ \\ ${ }^{1}$ Department of Mathematics, University College of Qunfudha, Umm Alqura University, Makkah, KSA \\ ${ }^{2}$ Engineering College, Albaha University, Albaha, KSA
}

Email address:

samialtoum@hotmail.com (S. H. Altoum), salihyou@gmail.com (S. Y. Arbab)

\section{To cite this article:}

Sami H. Altoum, Salih Y. Arbab. Comparison Solutions Between Lie Group Method and Numerical Solution of (RK4) for Riccati Differential Equation. Applied and Computational Mathematics. Vol. 5, No. 2, 2016, pp. 64-72. doi: 10.11648/j.acm.20160502.15

Received: February 17, 2016; Accepted: March 25, 2016; Published: April 15, 2016

\begin{abstract}
This paper introduced Lie group method as a analytical method and then compared to RK4 and Euler forward method as a numerical method. In this paper the general Riccati equation is solved by symmetry group. Numerical comparisons between exact solution, Lie symmetry group and RK4 on these equations are given. In particular, some examples will be considered and the global error computed numerically.
\end{abstract}

Keywords: Riccati Equation, Symmetry Group, Infinitesimal Generator, Runge-Kutta

\section{Introduction}

$$
f(x, y)=P(x)+Q(x) y+R(x) y^{2}
$$

where $\mathrm{P}(\mathrm{x}), \mathrm{Q}(\mathrm{x}), \mathrm{R}(\mathrm{x})$ functions of $\mathrm{x}$

The Riccati differential equation is named after the Italian nobleman Count Jacopo Francesco Riccati (1676-1754). The book of Reid [1] contains the fundamental theories of Riccati equation. This equation is perhaps one of the simplest nonlinear first ODE which plays a very important role in solution of various non-linear equations may be found in numerous scientific fields. The solution of this equation can be obtained numerically by using classical numerical method such as the forward Euler method and Runge-Kutta method. An analytic solution of the non-linear Riccati equation reached see [2] using A domain Decomposition Method. [5] used differential Transform Method(DTM) to solve Riccati differential equations with variable co-efficient and the results are compared with the numerical results by (RK4) method.[14] solved Riccati differential equations by using (ADM) method and the numerical results are compared with the exact solutions. B.Batiha [15] solved Riccati differential equations using Variational Iteration Method (VIM) and numerical comparison between VIM, RK4 and exact solution on these equations are given. Lie symmetries are utilized to solve both linear and non-linear first order ODE, and the majority of ad hoc methods of integration of ordinary differential equation could be explained and deduced simply by means of the theory of Lie group. Moreover Lie gave a classification of ordinary differential equation in terms of their symmetry groups. Lie's classification shows that the second order equations integrable by his method can be reduced to merely four distinct canonical forms by changes of variables. Subjecting these four canonical equations to changes of variables alone, one obtains all known equations integrated by classical methods as well as infinitely many unknown integrable equations. We will consider in our test only a first order differential equation and the numerical solution to second-order and higher-order differential equations is formulated in the same way as it is for first-order equations. We have attempted to give some examples to the use of Lie group methods for the solution of first-order ODEs. The Lie group method of solving higher order ODEs and systems of differential equations is more involved, but the basic idea is the same: we find a coordinate system in which the equations are simpler and exploit this simplification.

Definition: A change of variables, $(\mathrm{x}, \mathrm{y}) \rightarrow(\overline{\mathrm{x}}, \overline{\mathrm{y}})$, is called an equivalence transformation of Riccati differential equation if any equation of the form (1) transformed into an 
equation of same type with possibly different coefficients. Equations related by an equivalence transformation are said to be equivalent.

Theorem: The general equivalence transformation of Riccati equation comprises of

i. Change of independent variable $\bar{x}=\varphi(x), \varphi^{\prime}(x) \neq 0$

ii. Linear-rational transformations of the dependent variable

$$
\bar{y}=\frac{\alpha(x) y+\beta(x)}{\gamma(x) y+\delta(x)}, \alpha \delta-\beta \gamma \neq 0
$$

Example: Find the transformation of the coefficients of the Riccati equation under change of independent variable (i)

Solution: According to the chain rule of differentiation,

$$
\begin{gathered}
\frac{d y}{d x}=\varphi^{\prime}(x) \frac{d y}{d \bar{x}} \\
\varphi^{\prime}(x) \frac{d y}{d \bar{x}}=P(x)+Q(x) y+R(x) y^{2} .
\end{gathered}
$$

Upon division by $\varphi^{\prime}(\mathrm{x})$ and substitution $\mathrm{x}=\varphi^{-1}(\overline{\mathrm{x}})$, it becomes

$$
\frac{\mathrm{dy}}{\mathrm{dx}}=\overline{\mathrm{P}}(\overline{\mathrm{x}})+\overline{\mathrm{Q}}(\overline{\mathrm{x}}) \mathrm{y}+\overline{\mathrm{R}}(\overline{\mathrm{x}}) \mathrm{y}^{2}
$$

where

$$
\begin{gathered}
\bar{P}(\bar{x})=\frac{P\left(\varphi^{-1}(\bar{x})\right)}{\varphi^{\prime}\left(\varphi^{-1}(\bar{x})\right)}, \bar{Q}(\bar{x})=\frac{Q\left(\varphi^{-1}(\bar{x})\right)}{\varphi^{\prime}\left(\varphi^{-1}(\bar{x})\right)}, \\
\bar{R}(\bar{x})=\frac{R\left(\varphi^{-1}(\bar{x})\right)}{\varphi^{\prime}\left(\varphi^{-1}(\bar{x})\right)}
\end{gathered}
$$

\section{General Solution of Riccati Equation}

The general solution $\mathrm{y}(\mathrm{x})$ of equation (1), we suppose $\mathrm{s}(\mathrm{x})$ is a particular solution for (1) and also assume the general solution is

$$
y(x)=s(x)+\frac{1}{z(x)}
$$

where $\mathrm{z}(\mathrm{x})$ is unknown function and we seek about it by substitution the general solution $y(x)$ from (2) in (1) we deduce:

$$
s^{\prime}(x)-\frac{1}{z^{2}(x)} z^{\prime}(x)=P(x) s^{2}(x)+Q(x) s(x)+R(x)+P(x)\left(\frac{1}{z^{2}(x)}+\frac{2 s(x)}{z(x)}\right)+\frac{Q(x)}{z(x)}
$$

The particular solution $\mathrm{s}(\mathrm{x})$ is solution of (1) so satisfying it, consequently substitute instead of y in equation (1) we, get

$$
s^{\prime}(x)=P(x) s^{2}+Q(x) s(x)+R(x),
$$

so by substitution (4) in (3) we deduce:

$$
z^{\prime}(x)+[2 P(x) s(x)+Q(x)] z(x)=-P(x)
$$

It's clear equation (5) is linear equation from first order after solution we obtain $\mathrm{y}(\mathrm{x})$ and by choosing particular $\mathrm{s}(\mathrm{x})$ via (Trial and Error) we obtain the general solution

$$
y(x)=s(x)+\frac{1}{z(x)}
$$

\section{Solution of Lie Group Method O.D.E}

A set $G$ of invertible point transformation in the $(x, y)$ plane $\mathrm{R}^{2}$

$$
\bar{x}=f(x, y, a), \bar{y}=g(x, y, a),
$$

depending on a parameter $\mathrm{a}$ is called one-parameter continuous group, if $\mathrm{G}$
Contains the identity transformation (e.g. $f=x, g=y$ at $\mathrm{a}=0$ ) as well as the inverse of elements and their composition. It's said that transformations (6) form asymmetry group of differential equation

$$
\mathrm{F}\left(\mathrm{x}, \mathrm{y}, \mathrm{y}^{\prime}, \ldots, \mathrm{y}^{(\mathrm{n})}\right)=0
$$

if the equation is form invariant;

$$
\overline{\mathrm{F}}\left(\overline{\mathrm{x}}, \overline{\mathrm{y}}, \overline{\mathrm{y}}^{\prime}, \ldots, \overline{\mathrm{y}}^{(\mathrm{n})}\right)=0
$$

Whenever $\mathrm{F}\left(\mathrm{x}, \mathrm{y}, \mathrm{y}^{\prime}, \ldots, \mathrm{y}^{(\mathrm{n})}\right)=0$. A symmetry group of differential equation is also termed a group admitted by this equation. Lie's theory reduced the construction of the largest symmetry group $\mathrm{G}$ to the determination of it's infinitesimal transformations:

$$
\bar{x}^{i} \approx x+a \xi^{i}(x, y), \bar{y}^{i} \approx y+a \eta^{i}(x, y) .
$$

Where

$$
\xi^{i}(x)=\frac{\partial}{\partial a}\left[f^{i}(x, a)\right]_{a=0} .
$$

defined as a linear part (in the group parameter a ) in the Taylor expansion of the finite transformations (6) of G. It's convenient represent infinitesimal transformation (8) by the 
linear differential operator

$$
X=\xi^{i}(x, y) \frac{\partial}{\partial x}+\eta^{i}(x, y) \frac{\partial}{\partial y} .
$$

Called the infinitesimal transformation or infinitesimal operator of the group $\mathrm{G}$ with symmetry condition

$$
\eta_{\mathrm{x}}-\xi_{\mathrm{y}} \mathrm{h}^{2}+\left(\eta_{\mathrm{y}}-\xi_{\mathrm{x}}\right) \mathrm{h}-\left(\xi_{\mathrm{x}}+\eta \mathrm{h}_{\mathrm{y}}\right)=0
$$

The generator $\mathrm{X}$ of the group admitted by differential equation is also termed an operator admitted by this equation. An essential feature of a symmetry group $G$ is that it conserves the set of solutions of the differential equation admitting this group. Namely, the symmetry transformations merely permute the integral curves among themselves. It may happen that some of the integral curves are individually unaltered under group $\mathrm{G}$. Such integral curves are termed invariant solutions

\section{Canonical Variables}

Equation (7) furnishes us with a simple example for exhibiting the symmetry of differential equations. Since this equation does not explicitly contain the independent variable $\mathrm{x}$, it does not alter after any transformation $\bar{x}=\mathrm{x}+\mathrm{a}$ with an arbitrary parameter $\mathrm{a}$. The latter transformation from a group known as the group of translation along the $\mathrm{x}$-axis . Equation (7) is in fact the general nth-order ordinary differential equation admitting the group of translations along the $\mathrm{x}-$ axis. Moreover, any one parameter group reduces in proper variables to the group of translations. These new variables, canonical variables $\mathrm{t}$ and $\mathrm{u}$ are obtained by solving the equation

$$
X(t)=1, X(u)=0
$$

Where $\mathrm{X}$ is the generator (9) of group G. It follows that an nth-order ordinary differential equation admitting a oneparameter group reduces to the form (7) in the canonical variables, and hence one can reduce its order to $n-1$. In particular, any first-order equation with a known oneparameter symmetry group can be integrated by quadrature using canonical variables.

\section{Numerical Method}

We shall consider the solution of sets of first-order differential equations only. Users interested in solving higher order ODES can reduce their problem to a set of first order equations. Fortunately, the method of Lie group gives sometimes the solution of ODEs, but it's difficult to obtain an exact solution. For that, we will use numerical methods to obtain a solution. Since the numerical method used to compute an approximation at each step of the sequence, errors are compounded at every step. We introduce the numerical method will be compared to Lie group method and we don't forget the comparison of the error at each example will be presented. In this context, we will use Runge Kutta-4 and Euler forward methods which is the most widely one step methods to solve

$$
\frac{d}{d x} y(x, y)=f(x, y(x))
$$

involves defining 4 quantities $\mathrm{k}_{\mathrm{n}}$ as follows:

$$
\mathrm{k}_{\mathrm{n}}=\mathrm{f}\left(\alpha_{\mathrm{i}}+\mathrm{c}_{\mathrm{n}} \mathrm{h}, \mathrm{y}_{\mathrm{i}}+\mathrm{h} \sum_{\mathrm{m}=1}^{\mathrm{m}} \alpha_{\mathrm{n}, \mathrm{m}} \mathrm{k}_{\mathrm{m}}\right)
$$

here, $y_{i} \sim y\left(x_{i}\right)$ represents our numeric approximation to solution of the differential equation at $x=x_{i}$. The coefficients $\mathrm{y}_{\mathrm{i}} \sim \mathrm{y}\left(\mathrm{x}_{\mathrm{i}}\right)$ and $\mathrm{c}_{\alpha, \mathrm{m}}$ are constants that we are going to choose shortly, and $h=x_{i}=x_{i+1}-x_{i}$ is the step size. Once one calculates $\mathrm{k}_{\mathrm{n}}$ given at $\left(\mathrm{x}_{\mathrm{i}}, \mathrm{y}_{\mathrm{i}}\right)$ the value of $\mathrm{y}_{\mathrm{i}+1} \sim \mathrm{y}\left(\mathrm{x}_{\mathrm{i}+1}\right)$ is given by

$$
y_{i}=y_{i}+h \sum_{n=1}^{M} b_{n} k_{n}
$$

In this expression, the $b_{n}$ are constants.

$$
\begin{gathered}
\mathrm{k}_{1}=\mathrm{f}\left(\mathrm{x}_{\mathrm{i}}, \mathrm{y}_{\mathrm{i}}\right) \\
\mathrm{k}_{2}=\mathrm{f}\left(\mathrm{x}_{\mathrm{i}}+1 / 2 \mathrm{~h}, \mathrm{y}_{\mathrm{i}}+1 / 2 \mathrm{hk}_{1}\right) \\
\mathrm{k}_{3}=\mathrm{f}\left(\mathrm{x}_{\mathrm{i}}+1 / 2 \mathrm{~h}, \mathrm{y}_{\mathrm{i}}+1 / 2 \mathrm{hk}_{2}\right) \\
\mathrm{k}_{4}=\mathrm{f}\left(\mathrm{x}_{\mathrm{i}}+\mathrm{h}, \mathrm{y}_{\mathrm{i}}+\mathrm{hk}_{3}\right)
\end{gathered}
$$

Then we have

$$
\mathrm{y}_{\mathrm{i}+1}=\mathrm{y}_{\mathrm{i}}+\mathrm{h}\left(\frac{1}{6} \mathrm{k}_{1}+\frac{1}{3} \mathrm{k}_{2}+\frac{1}{3} \mathrm{k}_{3}+\frac{1}{6} \mathrm{k}_{4}\right)
$$

We recall that using $M=4$, (RK4) the global error is $\mathrm{O}\left(\mathrm{h}^{4}\right)$.

\section{Exact and Numerical Solution}

In this section we solve the following examples by two methods, firstly symmetry group solution and secondly numerically-forward Euler method and Runge-Kutta methods. Finally we see the privilege and more effective of mentioned methods.

\section{Example 1:}

First: Symmetry solution

Consider Riccati equation

$$
\frac{d y}{d x}+y^{2}-\frac{2}{x^{2}}=0 .
$$

In this example we see the function is not define in $\mathrm{x}=0$. This equation admits the one-parameter group of non- 
homogeneous dilations (scaling transformation) $\bar{x}=\mathrm{xe}^{\mathrm{a}}$, $\overline{\mathrm{y}}=\mathrm{ye}^{\mathrm{a}}$. Indeed the derivative $\mathrm{y}^{\prime}$ is written in the new variables as $\overline{\mathrm{y}}^{\prime}=\mathrm{y}^{\prime} \mathrm{e}^{-\mathrm{a}}$, whence

$$
\frac{d \bar{y}}{d \bar{x}}+\bar{y}-\frac{2}{\bar{x}^{2}}=\left(\frac{d y}{d x}+y^{2}-\frac{2}{x^{2}}\right) e^{-a}=0 \text {. }
$$

Hence, the equation is form invariant. The symmetry group has the generator

$$
X=x \frac{\partial}{\partial x}+y \frac{\partial}{\partial y}
$$

The solution of the equation with the above operator $\mathrm{X}$ provides canonical variables, from equation (11) we, get

$$
\mathrm{t}=\ln (\mathrm{x}) \text { and } \mathrm{u}=\mathrm{xy} \text {. }
$$

In these variables, the original Riccati equation takes the following integrable form:

$$
\frac{\mathrm{du}}{\mathrm{dt}}+\mathrm{u}^{2}-\mathrm{u}-2=0
$$

Then we, get the final solution of equation (13) is

$$
y=-\frac{1}{x} \text { and } y=\frac{2}{x} .
$$

\section{Second: Classical Solution}

Here we using given initial condition $y(1)=-1$. If $y=\frac{2}{x}$ is a particular solution, then $y=u+\frac{2}{x}$ is the general solution. Satisfies the Bernoulli equation

$$
u^{\prime}+\frac{4}{x} u=-u^{2}
$$

On the natural way, denote further $\mathrm{v}=\mathrm{u}^{-1}$ to obtain the linear equation

$$
\mathrm{vc}-\frac{4}{\mathrm{x}} \mathrm{v}=1
$$

Now we derive the solution of the linear equation

$$
\mathrm{v}=\frac{\mathrm{x}\left(3 \mathrm{cx^{3 } - 1 )}\right.}{3} \quad 3 \mathrm{c}=\mathrm{c}_{1} \text { cons tan ts }
$$

So the solution is $y=\frac{-1+2 c_{1} x^{3}}{x\left(1+c_{1} x^{3}\right)}$, if $c_{1}=0$ then the general solution $\mathrm{y}=-\frac{1}{\mathrm{x}}$.

Figure 1(a) give the behavior of the solution using RK4, Forward Euler and Lie group technique. We notice that there is a big error occurs using forward Euler. Moreover Figure 1(b), Figure 1(c) and Figure 1(d) confirm our predictions that there is a small error between analytical and numerical solution using RK4 but there is no-convergence when one use Forward Euler method.

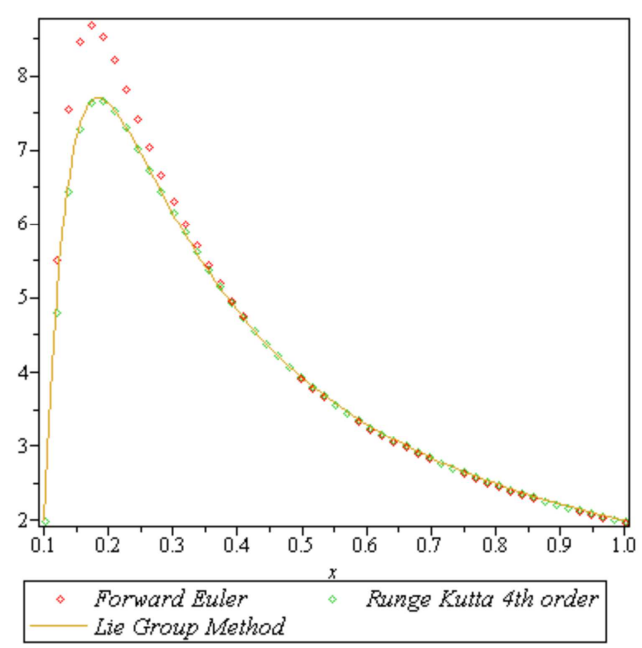

Fig. 1(a). Case $N=50$.

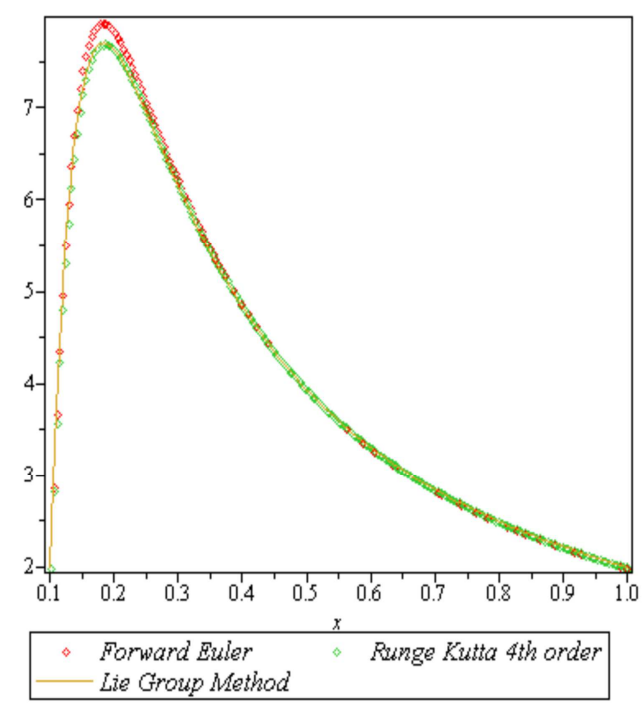

Fig. 1(b). Case $N=200$.

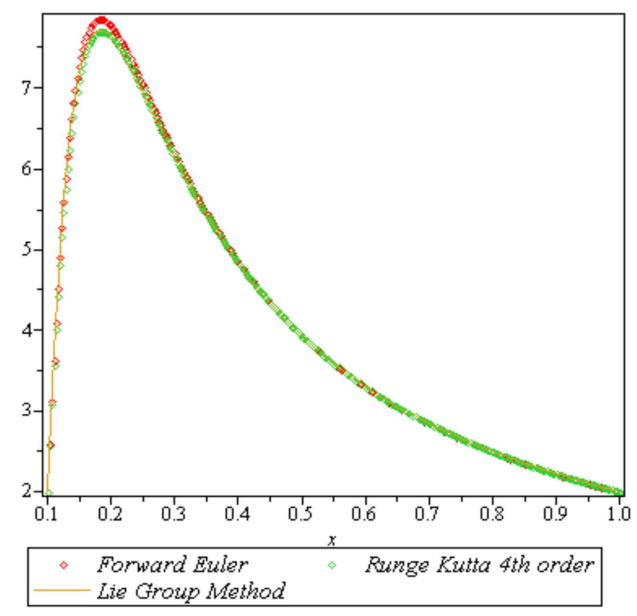

Fig. 1(c). Case $N=300$. 


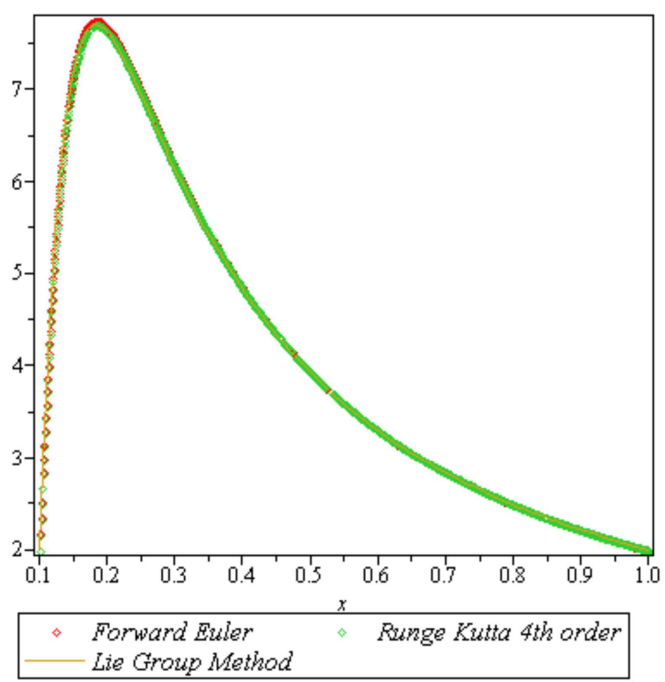

Fig. $1(d)$. Case $N=900$.

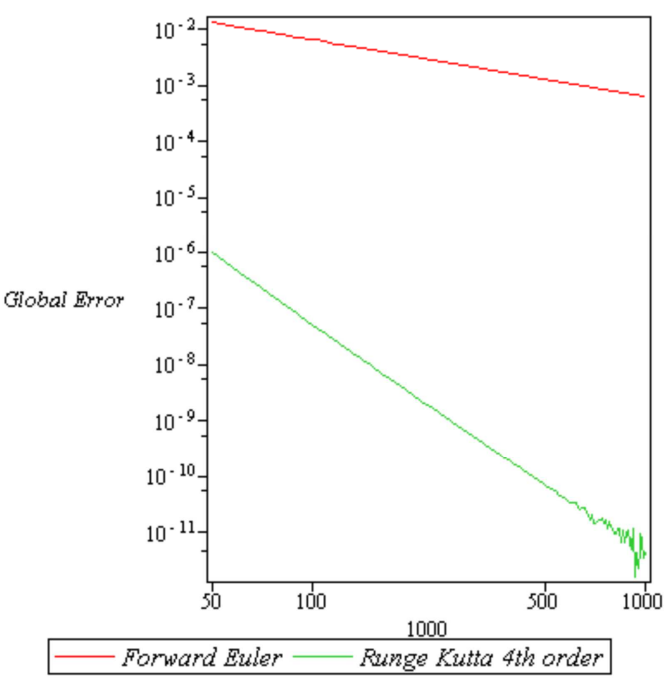

Fig. 1. Solution and error of first order ode.

Table 1. Error between RK4 and Euler forward.

\begin{tabular}{llllllllll}
\hline $\mathbf{N}$ & $\mathbf{5 0}$ & $\mathbf{1 0 0}$ & $\mathbf{3 0 0}$ & $\mathbf{4 0 0}$ & $\mathbf{5 0 0}$ & $\mathbf{6 0 0}$ & $\mathbf{7 0 0}$ & $\mathbf{8 0 0}$ & $\mathbf{9 0 0}$ \\
\hline$\varepsilon_{\text {Euler }}$ & $1.35 \mathrm{E}-2$ & $6.63 \mathrm{E}-3$ & $2.17 \mathrm{E}-3$ & $1.62 \mathrm{E}-3$ & $1.12 \mathrm{E}-3$ & $1.10 \mathrm{E}-3$ & $9.26 \mathrm{E}-4$ & $8 \mathrm{E}-4$ & $7 \mathrm{E}-4$ \\
$\varepsilon_{\text {RK } 4}$ & $9.9 \mathrm{E}-7$ & $5.15 \mathrm{E}-8$ & $5.60 \mathrm{E}-10$ & $1.74 \mathrm{E}-10$ & $7.08 \mathrm{E}-11$ & $3.38 \mathrm{E}-11$ & $1.44 \mathrm{E}-11$ & $1.13 \mathrm{E}-11$ & $6.41 \mathrm{E}-12$ \\
\hline
\end{tabular}

We see for even a moderate number of steps, the agreement between the Runge-Kutta method and the analytic solution is remarkable. We can quantify just how much better the Runge-Kutta stencil does by defining a measure of the global error $\varepsilon$ as the

$$
\mathcal{E}^{k}\left(y_{0}, N, \text { Method }\right)=\left|\operatorname{Method}\left(y_{0} N\right)-y_{L G}^{k}\right|_{x=1} \mid
$$

Where the method $\in\{$ Euler, RK 4$\}, \mathrm{k}=1,2,3$. Clearly, the error for the Runge-Kutta method is several orders of magnitude lower. Furthermore, the global error curves both look linear on the log-log plot, which suggests that there is a power law dependence of $\varepsilon$ on

$$
N: \mathcal{E} \sim \varepsilon_{0} N^{-\alpha} \alpha h^{\alpha}
$$

Example 2: Consider the Riccati equation

$$
y^{\prime}=x y^{2}-\frac{2 y}{x}-\frac{1}{x^{3}}
$$

corresponding to the choice $\mathrm{R}(\mathrm{x})=\mathrm{x}, \mathrm{Q}(\mathrm{x})=\frac{-2}{\mathrm{x}}, \mathrm{P}(\mathrm{x})=\frac{-1}{\mathrm{x}^{3}}$. In equation (1) Show that the transformation $X=x \frac{\partial}{\partial x}-2 y \frac{\partial}{\partial y}$ is a one parameter Lie group of scaling symmetries for this Riccati equation. Use the one parameter Lie group to show that it has two invariant solutions

$$
y= \pm x^{2}
$$

Use the method of characteristics to solve the defining Eqs. (11) for canonical coordinates. Show that

$$
(t, u)=\left(x^{2} y, \ln |x|\right)
$$

is a solution for $\mathrm{x} \neq 0$.

Show that in these canonical coordinates the Riccati Eq. (14) reduces to

$$
\frac{\mathrm{du}}{\mathrm{dt}}=\frac{1}{\mathrm{t}^{2}-1}
$$

Integrate the last equation to show that in the original $(\mathrm{x}, \mathrm{y})$ coordinates,

$$
\begin{gathered}
\frac{\mathrm{du}}{\mathrm{dt}}=-\frac{1}{1-\mathrm{t}^{2}} \\
\mathrm{u}+\mathrm{c}=-\arctan (\mathrm{t})
\end{gathered}
$$

where c real parameter will be determinate using initial condition

Substitute $(t, u)=\left(x^{2} y, \ln |x|\right)$, we get

$$
\begin{gathered}
\ln |x|+c=-\tanh ^{-1}\left(x^{2} y\right) \\
-(\ln |x|+c)=\tanh ^{-1}(x y)
\end{gathered}
$$

For $\mathrm{x}$ different to zero we have

$$
\begin{aligned}
& \frac{1}{x^{2}} \tanh (-\ln |x|+c)=y \\
& y=-\frac{1}{x^{2}} \tanh (-\ln |x|+c)
\end{aligned}
$$

Using $\mathrm{y}(0.1)=2$, we can determine the value of $\mathrm{c}$ 


$$
-\frac{1}{(0.1)^{2}} \tanh (\ln |\mathrm{x}|+\mathrm{c})=2
$$

$\tanh (\ln |\mathrm{x}|+\mathrm{c})=-2(0.1)^{2}=-0.02$

$$
\begin{aligned}
& \ln |\mathrm{x}|+\mathrm{c}=\arctan \mathrm{h}(-0.02) \\
& \mathrm{c}=\arctan (-0.02)-\ln \left(\frac{1}{10}\right)
\end{aligned}
$$

using $\arctan (-x)=-\arctan (x)$ we, get

$$
\mathrm{c}=\ln (10)+\arctan \left(-\frac{1}{50}\right)
$$

Finally the solution is

$$
y=-\frac{\tanh \left(\ln (x)+\ln (10)-\arctan h\left(\frac{1}{50}\right)\right)}{x^{2}}
$$

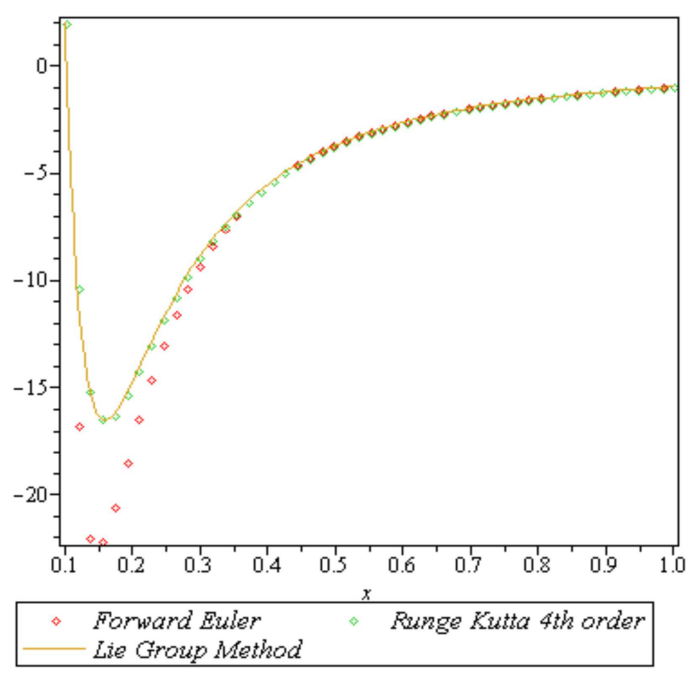

Fig. 2(a). Solution RK4 and Lie Group case $N=50$.

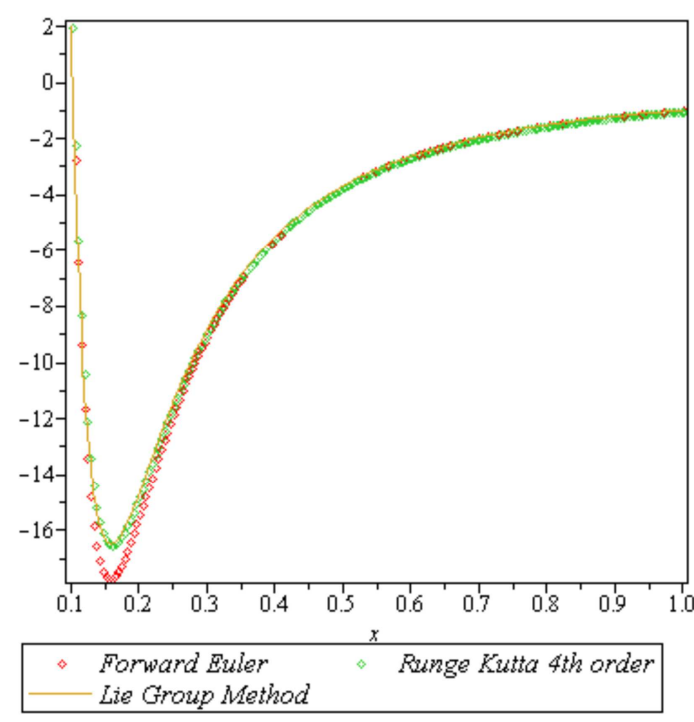

Fig. 2(b). Solution RK4 and Lie Group case $N=200$.

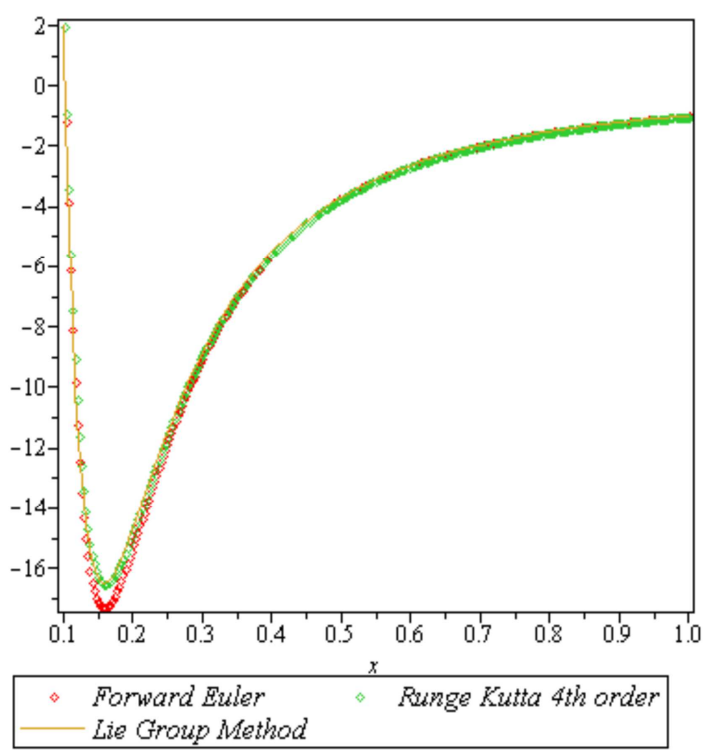

Fig. 2(c). Solution RK4 and Lie Group case N=300.

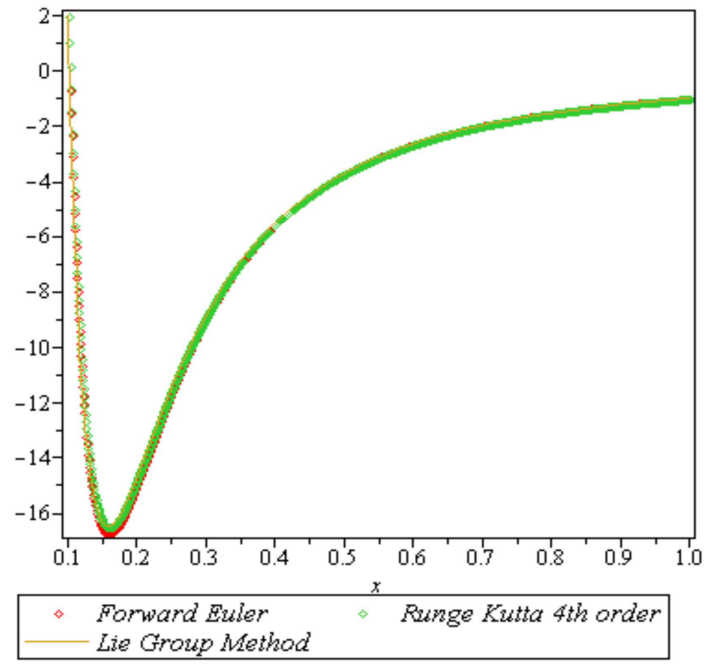

Fig. 2(d). Solution RK4 and Lie Group case N=900.

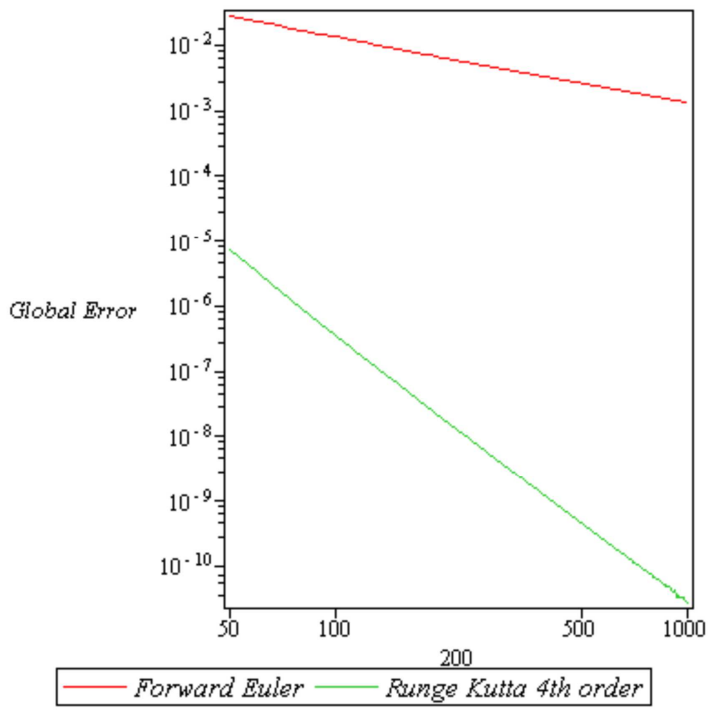

Fig. 2. Global Error 
Table 2. Error between RK4 and Euler forward.

\begin{tabular}{llllllllll}
\hline $\mathbf{N}$ & $\mathbf{5 0}$ & $\mathbf{1 0 0}$ & $\mathbf{3 0 0}$ & $\mathbf{4 0 0}$ & $\mathbf{5 0 0}$ & $\mathbf{6 0 0}$ & $\mathbf{7 0 0}$ & $\mathbf{8 0 0}$ & $\mathbf{9 0 0}$ \\
\hline$\varepsilon_{\text {Euler }}$ & $2.73 \mathrm{E}-2$ & $1.33 \mathrm{E}-2$ & $4.3 \mathrm{E}-3$ & $3.2 \mathrm{E}-3$ & $2.5 \mathrm{E}-3$ & $2.1 \mathrm{E}-3$ & $1.8 \mathrm{E}-3$ & $1.6 \mathrm{E}-3$ & $1.4 \mathrm{E}-3$ \\
$\varepsilon_{\text {RK } 4}$ & $67.98 \mathrm{E}-6$ & $3.41 \mathrm{E}-7$ & $3.90 \mathrm{E}-9$ & $1.11 \mathrm{E}-9$ & $4.53 \mathrm{E}-10$ & $2,14 \mathrm{E}-10$ & $1.13 \mathrm{E}-10$ & $6.96 \mathrm{E}-11$ & $4.48 \mathrm{E}-11$ \\
\hline
\end{tabular}

Example 3 Consider the Bernoulli equation $\mathrm{y}^{\prime}=\mathrm{y}+\mathrm{y}^{-1} \mathrm{e}^{\mathrm{x}}$, when substituted into condition (10), leads to

$$
\eta_{\mathrm{x}}-\xi_{\mathrm{y}}\left(\mathrm{y}+\mathrm{y}^{-1} \mathrm{e}^{\mathrm{x}}\right)^{2}+\left(\eta_{\mathrm{y}}-\xi_{\mathrm{x}}\right)\left(\mathrm{y}+\mathrm{y}^{-1} \mathrm{e}^{\mathrm{x}}\right)-\left(\xi\left(\mathrm{y}^{-1} \mathrm{e}^{\mathrm{x}}\right)+\eta\left(1-\mathrm{e}^{\mathrm{x}} / \mathrm{y}^{2}\right)\right)=0
$$

This, again, is too difficult as it sits, so we try a few simplifying assumptions before we discover that $\xi=1, \eta=\eta(\mathrm{y})$ yields

$$
\eta_{y}\left(y+y^{-1} e^{x}\right)+\left(y^{-1} e^{x}\right)-\eta\left(1-y^{-2} e^{x}\right)=0
$$

Because some terms depend only on $y$, we solve $y \eta_{y}-\eta=0$ to obtain $\eta=c y$. Inserting this form of 'into the remaining equation $\eta_{y}+y^{-1} \eta-1=0$, we arrive at $\eta=y / 2$. Now that we have settled on the symbols $(\xi, \eta)=(1, y / 2)$, we find canonical coordinates by solving $\frac{\mathrm{dy}}{\mathrm{dx}}=\frac{\eta}{\xi}=\frac{\mathrm{y}}{2}$ to get $\mathrm{u}$ and $\mathrm{t}$. Remember that we seek families of functions that remain constant for $\mathrm{u}$ so $\mathrm{u}=\mathrm{c}=\mathrm{ye}^{-\mathrm{x} / 2}$. The second coordinate $s$ is found by integrating $\mathrm{du}=\frac{\mathrm{dx}}{1}$ to get $\mathrm{u}=\mathrm{x}$. The next step is to find the differential equation in the canonical coordinates by computing;

$$
\frac{d u}{d t}=\frac{u_{x}+u_{y} h}{t_{x}+t_{y} h} .
$$

We learn that

$$
\frac{\mathrm{du}}{\mathrm{dt}}=\frac{1}{\frac{1}{2} \mathrm{ye}^{-\mathrm{x} / 2}+\mathrm{e}^{-\mathrm{x} / 2}\left(\mathrm{y}+\mathrm{y}^{-1} \mathrm{e}^{\mathrm{x}}\right)}=\frac{1}{\frac{1}{2} \mathrm{ye}^{-\mathrm{x} / 2}+\mathrm{y}^{-1} \mathrm{e}^{\mathrm{x} / 2}} .
$$

Expressing in $\frac{1}{2} \mathrm{ye}^{-\mathrm{x} / 2}+\mathrm{y}^{-1} \mathrm{e}^{\mathrm{x} / 2}$ terms of $\mathrm{t}$ and $\mathrm{u}$ leads to $\frac{\mathrm{t}}{2}+\frac{1}{\mathrm{t}}$, whence

$$
\frac{\mathrm{du}}{\mathrm{dt}}=\frac{\mathrm{t}}{\frac{\mathrm{t}^{2}}{2}+1}
$$

This integrates to

$$
\mathrm{u}=\ln \left(\frac{\mathrm{t}^{2}}{2}+1\right)+\mathrm{c}
$$

Returning to the original coordinates, we obtain

$$
\mathrm{x}=\ln \left(\frac{\mathrm{y}^{2} \mathrm{e}^{-\mathrm{x}}}{2}+1\right)+\mathrm{c}
$$

$$
\sqrt{2 \mathrm{e}^{\mathrm{x}}\left(\mathrm{e}^{(\mathrm{x}-\mathrm{c})}-1\right)}=\mathrm{y}
$$

Now from initial condition $\mathrm{y}(0.1)=2$ we, get

$$
\begin{gathered}
2 \mathrm{e}^{0.1}\left(\mathrm{e}^{0.1-\mathrm{c}}-1\right)=\mathrm{y} \\
\mathrm{e}^{0.1-\mathrm{c}}-1=\frac{4}{2} \mathrm{e}^{=0.1} \\
\mathrm{e}^{0.1-\mathrm{c}}=1+2 \mathrm{e}^{-0.1} \\
\mathrm{e}^{-\mathrm{c}}=\mathrm{e}^{-0.1}+2 \mathrm{e}^{-0.2} \\
\mathrm{c}=-\ln \left(\mathrm{e}^{-0.1}+2 \mathrm{e}^{-0.2}\right) \\
\mathrm{c}=-\ln \left(\mathrm{e}^{-0.2}\left(2+\mathrm{e}^{0.1}\right)\right) \\
\mathrm{c}=\frac{1}{5}-\ln \left(2+\mathrm{e}^{0.1}\right) \\
\mathrm{e}^{-\mathrm{c}}=\mathrm{e}^{-\frac{1}{5}}\left(2+\mathrm{e}^{0.1}\right)
\end{gathered}
$$

Finally we, get

$$
y=\sqrt{2 e^{x}\left(e^{x} e^{-\frac{1}{5}}\left(2+e^{0.1}\right)\right)-1}
$$
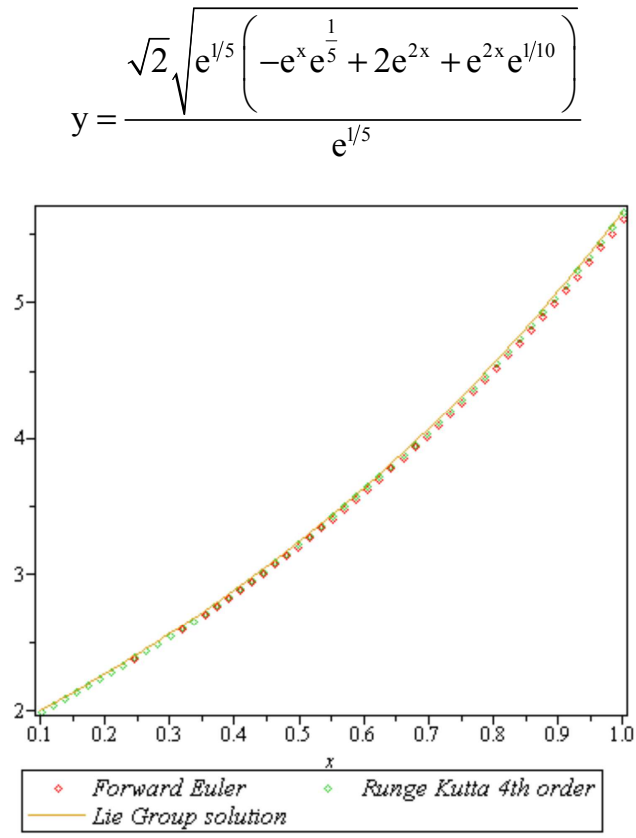

Fig. 3(a). Solution RK4 and Lie Group case $N=50$. 


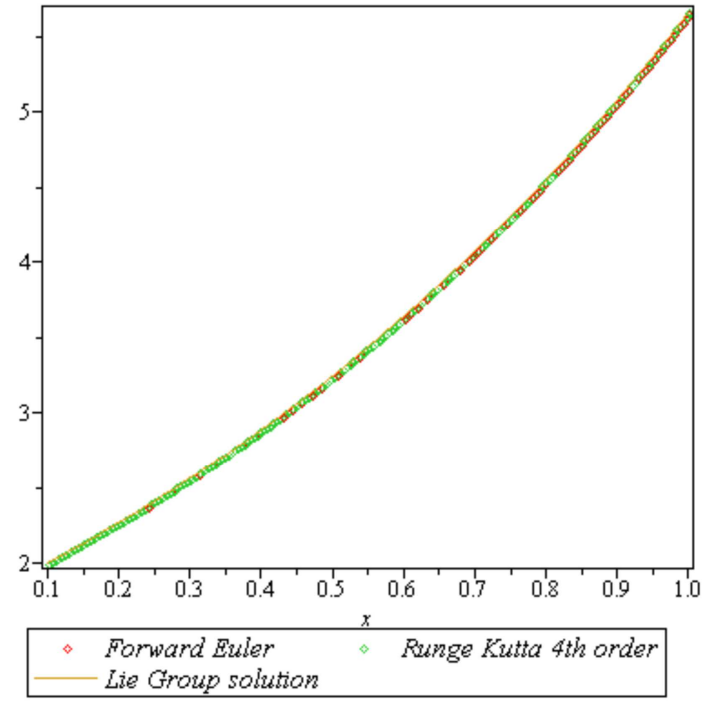

Fig. 3(b). Solution RK4 and Lie Group case $N=200$.

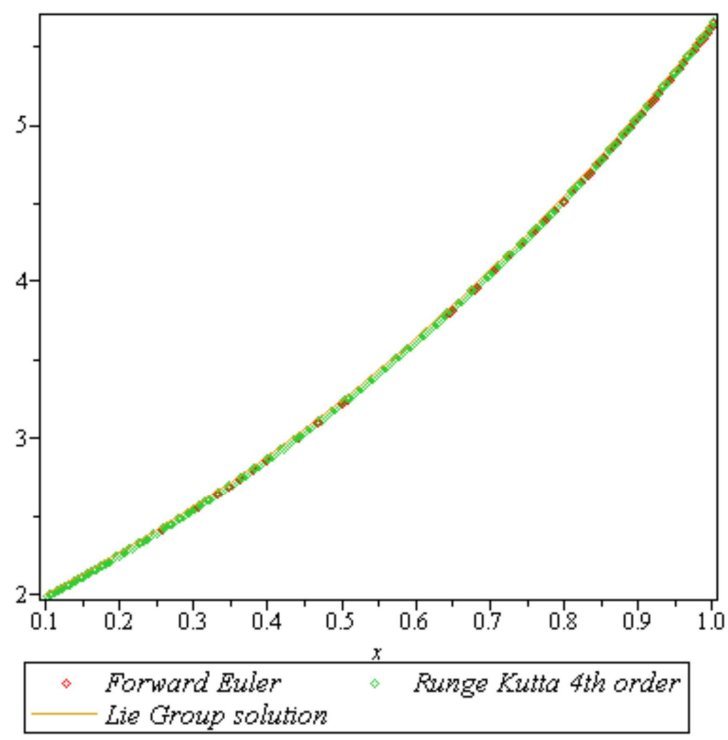

Fig. 3(c). Solution RK4 and Lie Group case $N=300$.

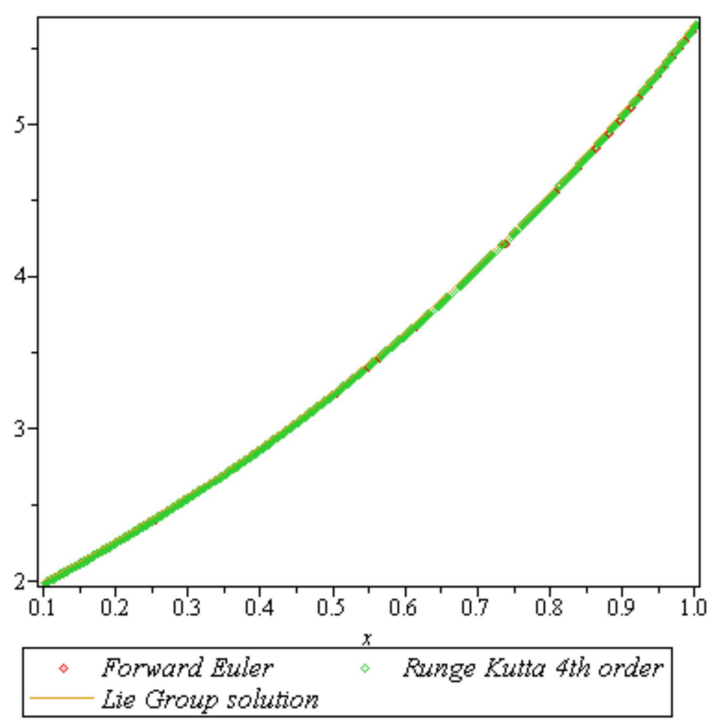

Fig. 3(d). Solution RK4 and Lie Group case $N=900$.

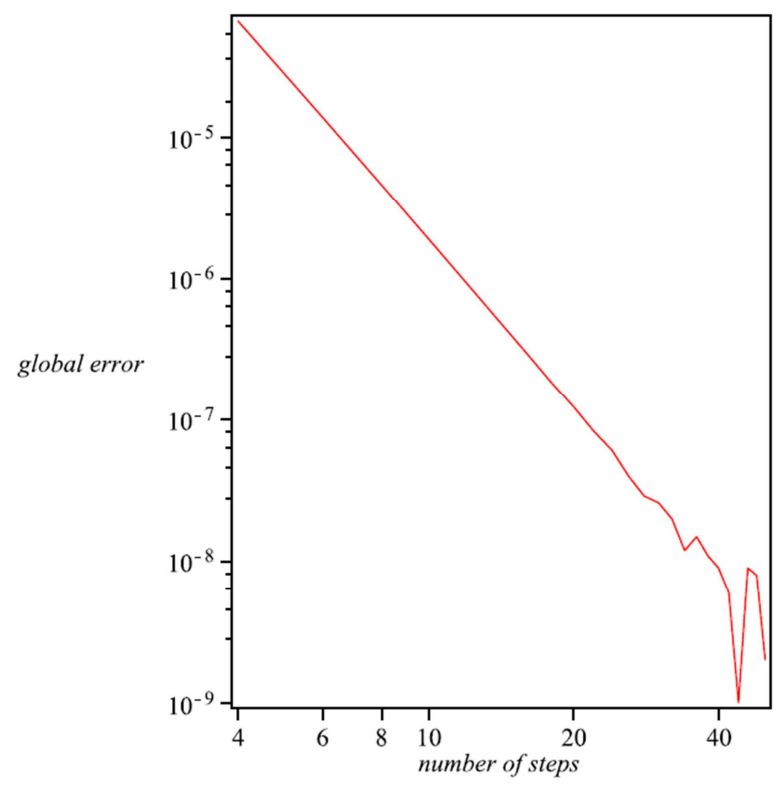

Fig. 3. Global Error

Here, we compare the solution of the Bernoulli equation obtained using Lie group and the numerical solution given by RK4, see Figure 3(a). We see for even a moderate number of steps, the agreement between the Runge- Kutta method and the analytic solution is remarkable. We can quantify just how much better the Runge-Kutta method does by defining a measure of the global error $\mathrm{e}$ as the magnitude of the discrepancy between the numerical and actual values of $y(1)$, see Figure 3(b). Clearly, the error for the Runge-Kutta method is several orders of magnitude lower. Furthermore, the global error curves both look linear on the log-log plot, which suggests that there is a power law dependence of $\varepsilon$ on $\mathrm{N}$. We can determine the power a by fitting a power law to the data obtained in our Maple code this leads that the global error using RK4 is $\mathrm{O}\left(\mathrm{h}^{.3973507074}\right)$ this match our expectation that the one step error is $\mathrm{O}\left(\mathrm{h}^{5}\right)$.

\section{Conclusions}

In this work we conclude that, firstly the general solution of Riccati equation is facing the problem of (Trial and Error) and it is lead to the classical method. Secondly we used Numerical solution (Forward Euler, RK4) method and we find the Lie group method is better than classical method but it fail at the singularity. The numerical solution is accuracy than symmetry group solution and classical method. In the last, we note that the Euler method are tested numerically and we have not convergence and the determined the global error is about $\mathrm{O}\left(\mathrm{h}^{0.9467945525}\right)$ and this don't match our expectation. The graphs in this have been performed using Maple 18.

\section{References}

[1] W. T. Reid, Riccati Differential Equations (Mathematics in science and engineering), New York: Academic Press, 1972. 
[2] F. Dubois, A. Saidi, Unconditionally Stable Scheme for Riccati Equation, ESAIM Proceeding. 8(2000), 39-52.

[3] A. A. Bahnasawi, M. A. El-Tawil and A. Abdel-Naby, Solving Riccati Equation using Adomians Decomposition Method, App. Math. Comput. 157(2007), 503-514.

[4] T. Allahviraloo, Sh. S. Bahzadi. Application of Iterative Methods for Solving General Riccati Equation, Int. J. Industrial Mathematics, Vol. 4, ( 2012) No. 4, IJIM-00299.

[5] Supriya Mukherjee, Banamali Roy. Solution of Riccati Equation with Variable Co-efficient by Differential Transform Method, Int. J. of Nonlinear Science Vol.14, (2012) No.2, pp. 251-256.

[6] Taiwo, O. A., Osilagun J. A. Approximate Solution of Generalized Riccati Differential Equation by Iterative Decomposition Algorithm, International Journal of Engineering and Innivative Technology(IJEIT) Vol. 1(2012) No. 2, pp. 53-56.

[7] J. Biazar, M. Eslami. Differential Transform Method for Quadratic Riccati Differential Equation, vol. 9 (2010) No.4, pp. 444-447.

[8] Cristinel Mortici. The Method of the Variation of Constants for Riccati Equations, General Mathematics, Vol. 16(2008) No.1, pp. 111-116.
[9] B. Gbadamosi, O. adebimpe, E. I. Akinola, I. A. I. Olopade. Solving Riccati Equation using Adomian Decomposition Method, International Journal of Pure and Applied Mathematics, Vol. 78(2012) No. 3, pp. 409-417.

[10] Olever. P. J. Application of Lie Groups to Differential Equations. New York Springer-Verlag, (1993).

[11] Al Fred Grany. Modern Differential Geometry of Curves and Surfaces, CRC Press, (1998).

[12] Aubin Thierry. Differential Geometry, American Mathematical Society, (2001).

[13] Nail. H. Ibragimov, Elementry Lie Group Analysis and Ordinary Differential Equations, John Wiley Sons New York, (1996).

[14] T. R. Ramesh Rao, "The use of the A domain Decomposition Method for Solving Generalized Riccati Differential Equations" Proceedings of the $6^{\text {th }}$ IMT-GT Conference on Mathematics, Statistics and its Applications (ICMSA2010) Universiti Tunku Abdul Rahman, Kuala Lumpur, Malaysia pp. 935-941.

[15] B. Batiha, M. S. M. Noorani and I. Hashim, " Application of Variational Iteration Method to a General Riccati Equation" International Mathematical Forum, Vol.2, no. 56, pp. 2759$2770,2007$. 